\title{
Investigação das alterações na percepção temporal pela presença de altos níveis de
}

\section{dependência de smartphone}

Investigation of alterations in temporal perception due to the presence of high levels of smartphone dependence

Investigación de las alteraciones de percepción temporal por la presencia de altos niveles de adicción al smartphone

Luís Augusto Soares Castellon ORCID: https://orcid.org/0000-0003-2875-9652 Universidade Estadual da Paraíba, Brasil

E-mail: luisaugustocastellon@gmail.com

Jonathan Bento Cavalcanti

ORCID: https://orcid.org/0000-0003-4129-4722

Universidade Estadual da Paraíba, Brasil E-mail: jonathan.ifpb@gmail.com

Renato Américo Dantas Camilo de Souza ORCID: https://orcid.org/0000-0001-8841-0853 Universidade Estadual da Paraíba, Brasil E-mail: macrenato2010@gmail.com

Fábio Galvão Dantas

ORCID: https://orcid.org/0000-0002-2923-9926 Universidade Estadual da Paraíba, Brasil E-mail: fabiogalvaodantas@gmail.com

\begin{abstract}
Resumo
Objetivo: Investigou-se a correlação entre a construção da percepção do tempo e de altos níveis de dependência de Smartphone, em estudantes universitários de Campina Grande - PB. Metodologia: Estudo descritivo na área de psicologia cognitiva, de natureza quantitativa e experimental. A pesquisa foi realizada de forma virtual, na plataforma Google Forms, pela aplicação de I-) Questionário socioeconômico; II-) Escala de Dependência de Smartphone (EDS); III-) Apresentação de estímulo padronizado (466 segundos); IV-) Questionário sobre a percepção do estímulo. As variáveis foram analisadas no programa Statistical Package for the Social Sciences (SPSS) - versão 20.0. Resultados: Participaram da pesquisa 155 estudantes, sendo 115 do gênero feminino $(74,2 \%)$ e 40 do masculino $(25,8 \%)$, com a média de idade de 21,5 anos. Para fins deste artigo, comparou-se o grupo sem dependência com o que apresentou alto nível de dependência. Através da EDS, observou-se que 41 (26,45\%) dos participantes não apresentaram dependência de Smartphone e $33(21,29 \%)$ apresentaram alto nível. A estimativa média do grupo sem dependência foi de 409 s para o estímulo apresentado, enquanto a do grupo com alto nível de dependência foi de 523s (27,8\% maior). O teste exato de Fisher indica correlação significativa $\left(X^{2}(4)=20,373, p<0,001\right)$ entre a percepção da duração do tempo do estímulo e alto nível de dependência de Smartphone. Conclusão: O grupo com alto nível de dependência de Smartphone apresentou percepção aumentada de duração do tempo em relação ao grupo sem dependência.
\end{abstract}

Palavras-chave: Percepção temporal; Dependência de smartphone; Tecnologia.

\begin{abstract}
Objective: We aim to investigate the correlation between the construction of perception of time and smartphone dependence in university students in Campina Grande - PB. Methodology: This was a quantitative and experimental cognitive psychology study. Using Google Forms platform, we applied: I-) Socioeconomic Questionnaire; II-) Smartphone Dependence Scale (SDS); III-) Standardized stimulus presentation (466 seconds) and IV-) Questionnaire on stimulus perception. Variables were analyzed by the Statistical Package for Social Sciences (SPSS) - version 20.0. Results: 155 students were included, 115 female (74.2\%) and 40 male (25.8\%), average of 21.5 years old. Students were divided into two groups, according to SDS: $41(26.45 \%)$ had no smartphone dependency and $33(21.29 \%)$ were dependent. The perception of duration of the stimulus was 409 seconds among students without dependence, and 523 seconds among the others $(27.8 \%$ higher - was $409 \mathrm{~s}$ for the stimulus presented, while that of the group with a high level of dependence was 523s $(27.8 \%$ higher, $\mathrm{p}<0.001)$. Conclusion: Students who were dependent on smartphone had a greater perception of time duration compared to the group without dependence.
\end{abstract}

Keywords: Temporal perception; Smartphone dependency; Technology. 


\begin{abstract}
Resumen
Objetivo: Se investigó la correlación entre la construcción de la percepción del tiempo y de altos niveles de adicción al Smartphone, en estudiantes universitarios de Campina Grande - Paraíba. Metodología : Estudio descriptivo en el área de psicología cognitiva, de naturaleza cuantitativa y experimental. El sondeo fue realizado de manera virtual, en la plataforma Google Forms, por la aplicación de I-) Cuestionario socioeconómico; II-) Escala de dependencia del smartphone (EDS); III-) Presentación de estímulo estandarizado (466 segundos); IV-) Cuestionario sobre la percepción del estímulo. Las variables fueron analizadas por el programa Statistical Package for the Social Sciences (SPSS) versión 20.0. Resultados: Participaron de la pesquisa 155 estudiantes, siendo 115 de género feminino (74,2\%) y 40 de género masculino (25,8\%), con edad mediana de 21,5 años. Para fines de este estudio, se comparó el grupo sin adicción al que presentó alto nivel de adicción. A través de la EDS, se ha observado que $41(26,45 \%)$ de los participantes no han presentado adicción al Smartphone y $33(21,29 \%)$ han presentado alto nivel. La estimativa mediana del grupo sin adicción fue de 409s para el estímulo presentado, mientras que la del grupo con alto nivel de adicción fue de 523s $(27,8 \%$ mayor). El test exato de Fisher indica correlación significativa $\left(X^{2}(4)=20,373\right.$, p < 0,001) entre percepción de duración del tempo de estímulo y alto nivel de adicción a Smartphones. Conclusión: El grupo con alto nivel de adicción al Smartphone presentó percepción aumentada de duración del tiempo en relación al grupo sin adicción.
\end{abstract}

Palabras clave: Percepción temporal; Adicción al smartphone; Tecnología.

\title{
1. Introdução
}

Em um contexto de explosão tecnológica no século XXI, com aparelhos de Smartphone cada vez mais sofisticados, bem como o aumento do consumo de tecnologia para execução das tarefas diárias pela população geral, como o estudo, o trabalho, o entretenimento e contato social; novas formas psicopatológicas surgiram, como no caso das dependências comportamentais de tecnologia (Picon, Karam, Breda, Restano, Silveira, \& Spritzer, 2015). Somado-se a isso, com o advento da pandemia de COVID19; o mundo virtual, a internet e as redes sociais, tornaram-se refúgio para o entretenimento e o contato social entre os indivíduos durante a situação de isolamento e quarenta, de modo a endossar o aumento exponencial do uso aparelhos eletrônicos (Tejedor, Cervi, Pérez-Escoda, \& Tusa, 2020) e de propiciar um cenário para o desenvolvimento em larga escala de dependência de tecnologia, gerando impactos na saúde física e mental, com o aumento de ansiedade e depressão advindos da pandemia do coronavírus (Huckins, DaSilva, Wang, Hedlund, Rogers, Nepal, \& Campbell, 2020).

Em paralelo a isso, tem-se que os comportamentos aditivos podem ser caracterizados por comportamentos compulsivos em que se busca uma satisfação imediata, persistindo apesar das tentativas de interrompê-lo ou de não executá-lo pelo indivíduo, visando o alívio do desconforto, da tensão emocional, do humor negativo ou de sintomas de abstinência, apesar das consequências adversas (Tonelli, s.d). As alterações neuroplásticas decorrentes de comportamentos aditivos, como no uso de Smartphone, ocorrem pela reorganização dos circuitos de prazer e recompensa do cérebro, com mudanças no sistema dopaminérgico, de modo que as adições comportamentais podem se configurar de forma análoga aos transtornos aditivos provocados pelo uso de substâncias, gerando quadros complexos de dependência, impulsividade e compulsividade (Oliveira, 2012). A dependência de tecnologia se configura como a inabilidade do indivíduo de controlar o seu uso de tecnologia, havendo diversos subtipos, como dependência de jogos eletrônicos, internet, redes sociais, smartphones, pornografia on-line, etc; provocando efeitos negativos e prejuízo nas principais áreas da vida pessoal, com o comprometimento da saúde física e mental, das emoções, das relações interpessoais, do desempenho acadêmico ou profissional (Picon et al., 2015). O termo recentemente cunhado 'nomofobia' é utilizado para a sensação psicológica de mal-estar, desconforto e irritabilidade advindo de não estar conectado ao telefone móvel, criado a partir da junção dos termos, em inglês, "no mobile-phone phobia" (Bhattacharya, Bashar, Srivastava, \& Singh, 2019), sendo um sintoma extremo de altos níveis de dependência de Smartphone e de uso compulsivo do aparelho

Entende-se que a relação com os Smartphones tem impacto na cognição humana, de modo a provocar alterações na memória, na atenção e no sistema de gratificação do cérebro (Wilmer, Sherman, \& Chein, 2017), também envolvidos na percepção temporal. A mudança dos acontecimentos do mundo, naquilo que constatamos como "tempo", é um ponto de partida para as mais diversas filosofias e teorizações, de modo que a problemática do tempo pode ser dividida numa realidade em si 
mesma, na sua continuidade objetiva, e na conscientização ou percepção da passagem do tempo por um sujeito (Worms, 2004). O construto do tempo difere entre os indivíduos, sendo vivenciado de forma particular por cada um, a partir de inúmeras condições subjetivas que propiciam o processamento da duração dos estímulos, como as funções cognitivas de atenção, da memória e da percepção (Matthews \& Meck, 2016). Além disso, o tempo psicológico pode ser afetado pelas expectativas dos indivíduos em dado momento; pela dimensão emocional e artística (Ramos \& Bueno, 2012); pelo uso de substâncias psicotrópicas (Cheng, Ali \& Meck, 2007); por transtornos mentais (Allman, Yin \& Meck 2014), como a depressão (Kent, Van Doorn \& Klein, 2019), etc; sendo a quantidade e velocidade de estímulos processados na consciência um fator chave para entender esse construto (Glicksohn, 2001).

Desse modo, a percepção do tempo pode ser influenciada e manipulada a partir de fatores psicológicos, fisiológicos e da ordem sensorial, a depender da magnitude dos estímulos e do relógio interno dos indivíduos (Kononowicz, Van Rijn \& Meck, 2018), de modo que podem ocorrer sensações de dilatação e de fluidez temporal, conforme o aumento ou a diminuição da atividade cerebral e cognitiva (Glicksohn, 2001). A título ilustrativo das mudanças na ordem de percepção temporal, tem-se o "estado de flow" como um estado de alta performance, em que o indivíduo consegue aumentar substancialmente seu desempenho numa determinada atividade, mantendo-se em alto níveis de atenção focada e concentração, sem distratibilidade, sentindo enorme satisfação durante a execução na tarefa e sofrendo alterações na percepção da duração do tempo, que é percebido de forma fluida (Sinnett et al., 2020).

A partir disso, o presente estudo busca investigar a correlação entre a construção da percepção do tempo e suas alterações em decorrência dos altos níveis de dependência de Smartphone, considerando aspectos neuropsicológicos e cognitivos que possam levantar contribuições para a discussão desse fenômeno e seus possíveis impactos na saúde mental.

\section{Metodologia}

Este estudo possui caráter descritivo e exploratório na área de psicologia cognitiva, de natureza quantitativa e experimental (Estrela, 2018). O público-alvo dirigido foi o de estudantes do ensino superior público e privado, residentes de Campina Grande - PB, Brasil.

A execução da pesquisa ocorreu de forma virtual, através da plataforma Google Forms, após a concordância dos participantes com o Termo de Consentimento e Livre Esclarecido (TCLE), aprovado pelo Comitê de Ética de Pesquisa da Universidade Estadual da Paraíba (UEPB). O estudo foi realizado com a aplicação de três breves questionários e da apresentação de um estímulo padronizado para todos os participantes, sendo eles, na respectiva ordem, I-) Questionário socioeconômico; II-) Escala de Dependência de Smartphone (EDS); III-) Apresentação de estímulo padronizado; IV-) Questionário sobre a percepção do estímulo.

A Escala de Dependência em Smartphone (EDS) foi utilizada na pesquisa por apresentar uma consistência interna precisa e fidedigna, apontando um valor de 0,94 no coeficiente Alfa de Cronbach no estudo de validação do instrumento (Sales, Silva, Lopes \& Silva, 2018), permitindo verificar de forma segura os níveis de dependência de smartphone dos participantes. A escala é de ordem unifatorial. A dependência de Smartphone foi analisada através dos escores da EDS, com 14 perguntas de múltipla escolha, de escala do tipo Likert, respondida em 5 pontos de frequência acerca de comportamentos ligados à dependência, onde, 1 (nunca); 2 (raramente); 3 (às vezes); 4 (frequentemente) e 5 (muito frequentemente). Os escores de dependência foram resultantes da soma simples dos valores das respostas na escala Likert, variando de 14 a 70 pontos (Sales et al., 2018).

O estímulo apresentado aos participantes consistiu em uma tela que alternava entre cores, no formato de GIF, anexada ao formulário online. Nessa etapa, foi pedido aos estudantes que contassem 60 vezes a cor azul, quando aparecesse na tela. A cor azul se repetia num período de 7,7 segundos, de modo que a duração real da atividade foi de 466s (7 minutos e 46 segundos). Após a realização da atividade, os participantes responderam um questionário acerca da avaliação da duração e da percepção do 
tempo transcorrido (Deng, Kanthawala, Meng, Peng, Kononova, Hao, Zhang, \& David, 2019). As variáveis analisadas foram a percepção da duração, a estimativa do tempo do estímulo apresentado e a classificação hedônica do estímulo. A percepção de duração foi analisada numa escala Likert de 5 pontos, onde, 1 = o tempo passou muito rápido; 2 o tempo passou rápido; $3=0$ tempo passou de forma normal; 4 = o tempo demorou um pouco; 5 = o tempo demorou muito. A estimativa do tempo foi requisitada em minutos e segundos aos participantes, mas transformada e analisada somente em segundos. A classificação hedônica também foi respondida por meio de uma escala Likert, onde, 1 = muito entediante, $2=$ entediante, $3=$ médio, $4=$ parcialmente estimulante e $5=$ muito estimulante.

Os dados obtidos foram analisados no Statistical Package for the Social Sciences (SPSS) - versão 20.0. Para o EDS, obteve-se uma distribuição normal para os escores da amostra do presente estudo ( $\mathrm{p}>0,05)$, tornando-a ideal para categorização em quatro níveis de dependência a partir dos quartis dos escores advindos da EDS. As notas de corte foram: sem dependência (até 33 pontos), baixa dependência (de 34 a 41), dependência moderada (de 42 a 48) e alta dependência (de 49 a 70 ). Para fins deste artigo, foram comparados o grupo sem dependência e o grupo com altos níveis de dependência.

\section{Resultados}

A amostra total contou com um $n=155$, totalizando 115 participantes do gênero feminino $(74,2 \%)$ e 40 do masculino (25,8\%), com média de idade de 21,5 $\pm 4,24$ anos. Dentre os participantes, 64,5\% consideravam-se brancos, 27,7\% pardos e 7,1\% negros. Quanto à divisão dos grupos, 41 indivíduos compuseram o grupo sem dependência, enquanto 33 foram caracterizados

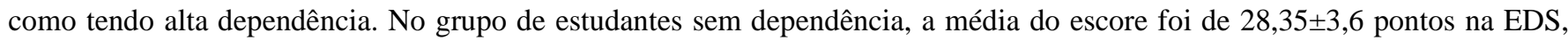
enquanto os indivíduos com alta dependência apresentaram um escore médio de 53,82 $\pm 4,1$ pontos. A distribuição de ambos os grupos pode ser observada no diagrama box-plot da Figura 1.

Figura 1: Diagramas box-plot comparando a distribuição dos scores na Escala de Dependência de Smartphone (EDS).

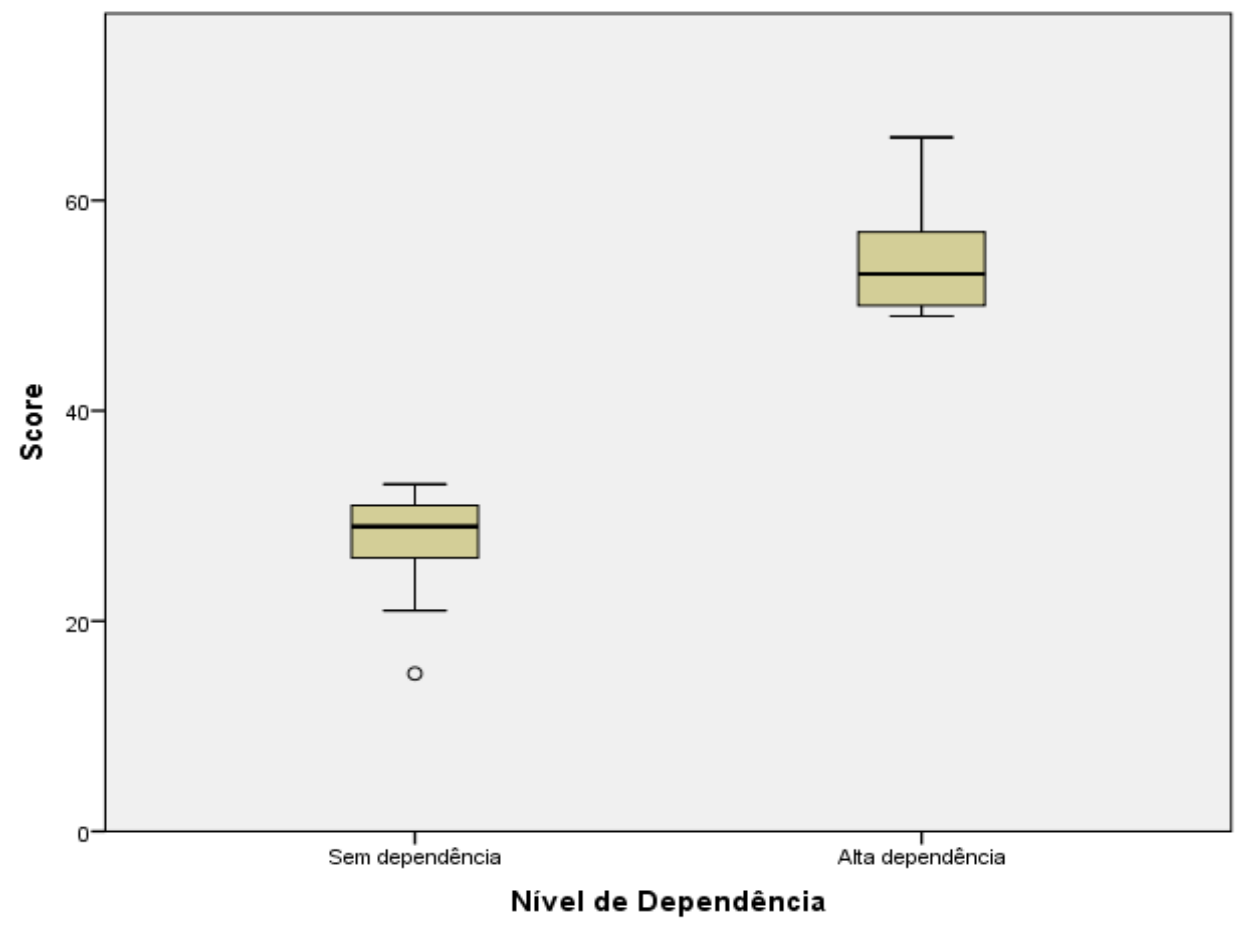

Fonte: Autores (2021). 


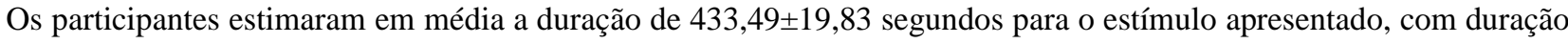
real de 466 segundos. A comparação das médias do tempo estimado pelos participantes do grupo sem e com alta dependência

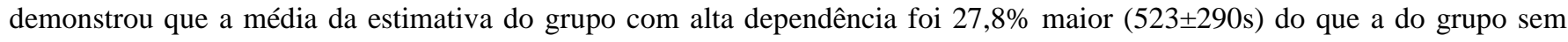

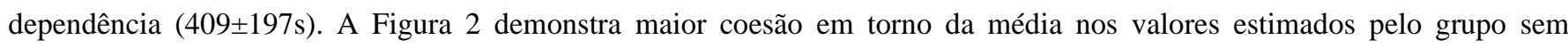
dependência, enquanto no grupo com alta dependência observou-se valores mais dispersos em torno da média. Nota-se apenas um outlier no grupo sem dependência e dois no grupo de alta dependência, embora em geral a dispersão em torno da média seja considerável em ambos.

Figura 2: Diagramas box-plot comparando a distribuição do tempo estimado entre os grupos sem dependência e com alta dependência em função dos valores das medianas, dos quartis e dos outliers observados.

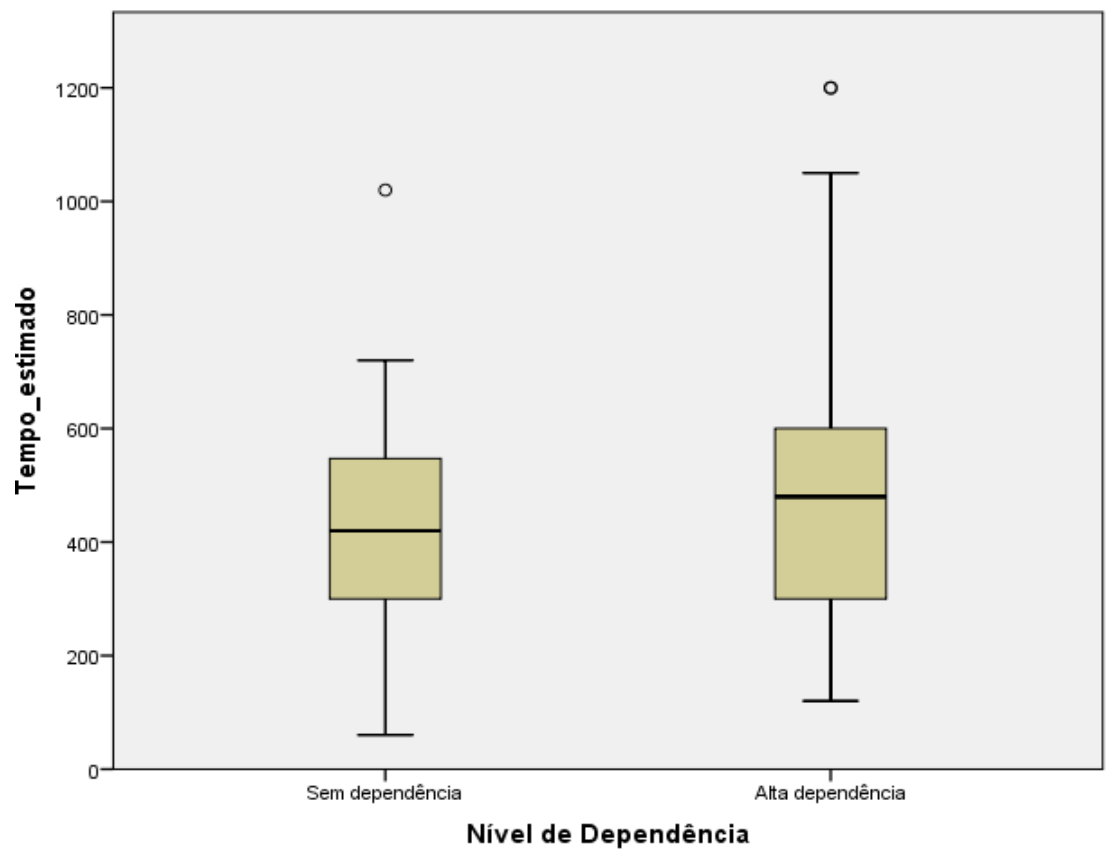

Fonte: Autores (2021).

A tabela da Figura 3 demonstra que a percepção de duração do estímulo também foi diferente, em detrimento do fator de alta dependência do Smartphone. O teste exato de Fisher mostrou que há associação entre a percepção da duração do tempo e ter ou não alta dependência do uso de smartphone $\left(\mathrm{X}^{2}(4)=20,373, \mathrm{p}<0,001\right)$. A estatística do V de Cramer indica que esta associação é de 50,6\% (p < 0,001), sendo relativamente alta. Pode-se observar que nenhum dos indivíduos com alta dependência considerou que o tempo do estímulo passou de forma normal, enquanto grande parte deste mesmo grupo achou que o tempo demorou muito $(66,7 \%)$. 
Figura 3: Tabela da distribuição da frequência da percepção da duração subjetiva do tempo entre os grupos sem dependência e com alta dependência.

\begin{tabular}{|c|c|c|c|c|c|}
\hline & & \multicolumn{4}{|c|}{ Nivel de Dependência } \\
\hline & & \multicolumn{2}{|c|}{$\begin{array}{c}\text { Sem } \\
\text { dependência }\end{array}$} & \multicolumn{2}{|c|}{$\begin{array}{c}\text { Alta } \\
\text { dependência }\end{array}$} \\
\hline & & $\mathrm{N}$ & $\%$ & $\mathrm{~N}$ & $\%$ \\
\hline \multirow[t]{6}{*}{ Duração Subjetiva } & $\begin{array}{l}\text { O tempo passou muito } \\
\text { rapido }\end{array}$ & 1 & $2,4 \%$ & 1 & $3,0 \%$ \\
\hline & O tempo passou rapido & 1 & $2,4 \%$ & 1 & $3,0 \%$ \\
\hline & $\begin{array}{l}\text { O tempo passou de } \\
\text { forma normal }\end{array}$ & 10 & $24,4 \%$ & 0 & $0,0 \%$ \\
\hline & $\begin{array}{l}\text { O tempo demorou um } \\
\text { pouco }\end{array}$ & 20 & $48,8 \%$ & 9 & $27,3 \%$ \\
\hline & O tempo demorou muito & 9 & $22,0 \%$ & 22 & $66,7 \%$ \\
\hline & Total & 41 & $100 \%$ & 33 & $100 \%$ \\
\hline
\end{tabular}

Fonte: Autores (2021).

Quanto à classificação hedônica — ou seja, o quão estimulante foi a atividade — a parcela maior dos participantes considerou o estímulo apresentado entediante, tanto para o grupo sem dependência (36,6\%) quanto para o grupo com alta dependência (45,5\%). As outras categorias da classificação hedônica também mostraram valores relativos bastante semelhantes, com exceção do número um pouco maior de participantes sem dependência que acharam o estímulo parcialmente estimulante $(14,6 \%)$. Percebe-se também que nenhum participante classificou o estímulo como muito estimulante. Todos os valores estão dispostos na tabela da Figura 4.

Figura 4: Tabela da distribuição da frequência hedônica do estímulo apresentado entre os grupos sem dependência e com alta dependência.

\begin{tabular}{|l|l|r|r|r|r|}
\hline \multicolumn{2}{|c|}{} & \multicolumn{4}{|c|}{ Nivel de Dependência } \\
\cline { 3 - 6 } \multicolumn{2}{|c|}{} & \multicolumn{2}{|c|}{ Sem dependência } & \multicolumn{2}{c|}{ Alta dependência } \\
\cline { 3 - 6 } & & $N$ & $\%$ & \multicolumn{1}{c|}{ N } & \multicolumn{1}{c|}{$\%$} \\
\hline \multirow{3}{*}{ Classificação Hedônica } & Muito entediante & 7 & $17,1 \%$ & 9 & $27,3 \%$ \\
\cline { 2 - 6 } & Entediante & 15 & $36,6 \%$ & 15 & $45,5 \%$ \\
\cline { 2 - 6 } & Médio & 13 & $31,7 \%$ & 6 & $18,2 \%$ \\
\cline { 2 - 6 } & Parcialmente Estimulante & 6 & $14,6 \%$ & 3 & $9,1 \%$ \\
\cline { 2 - 6 } & Muito estimulante & 0 & $0,0 \%$ & 0 & $0,0 \%$ \\
\cline { 2 - 6 } & Total & 41 & $100,0 \%$ & 33 & $100,0 \%$ \\
\hline
\end{tabular}

Fonte: Autores (2021).

\section{Discussão}

Ao longo das últimas décadas, diversos estudos de base experimental foram realizados com foco na produção de hipóteses e modelos teórico-metodológicos voltados à explicação dos aspectos subjetivos relacionados ao fenômeno de estimação do tempo em seres humanos, os quais poderiam ser categorizados em função de dois paradigmas de estimativa temporal: Paradigma Prospectivo, onde a atividade temporal a ser realizada pelos participantes da pesquisa lhes é revelada antes que o estímulo seja apresentado; Paradigma Retrospectivo, no qual a revelação da tarefa a ser executada é feita após a apresentação do estímulo (Zakay, 1990). A atividade proposta para verificar a percepção de duração do tempo seguiu um modelo retrospectivo, na qual os indivíduos descobriram posteriormente a finalidade da execução da tarefa, estimando um valor em 
minutos e segundos, bem como a impressão de quanto tempo durou, numa escala de "o tempo passou muito rápido" a "o tempo demorou muito" (Kent, Van Doorn \& Klein, 2019).

Melhor indicado para análises do processamento sensorial ligado à percepção da passagem do tempo, o modelo do Relógio Interno prediz que os mecanismos cerebrais ativados durante a exposição à estímulos externos que se repetem regularmente, sob a forma de pulsos, passam a diferir entre si a depender do tempo de duração destes mesmos estímulos (Block \& Zazay, 1996), ancorando-se na constatação de que haveria uma relação diretamente proporcional entre o acúmulo de pulsos percebidos e a indicação, pelos participantes, de estimativas temporais mais longas. Ou seja, enquanto que uma exposição de até 3 segundos envolveria predominantemente os circuitos neurais ligados à memória de trabalho (Rammsayer, Borter \& Troche, 2015), estímulos na faixa dos 4 segundos seriam predominantemente influenciados pelos sistemas de ativação do estado de alerta (Nather, Fernandes \& Bueno, 2014) e, acima dos 9 segundos, envolveriam mais intensamente as áreas da atenção (Droit-Volet, Monceau, Berthon, Trahanias \& Maniadakis, 2018). Considerando que o estímulo visual adotado na presente pesquisa utilizouse da alternância regular de cores, tal qual um pulso, com a atividade tendo duração total de 466 segundos (7 min 46 segundos), o modelo teórico supracitado parece se alinhar aos achados coletados à luz dos objetivos de análise propostos na presente pesquisa.

O intervalo de tempo de 7 min 46 segundos foi escolhido devido à aproximação do tempo em que os indivíduos de uso massivo de Smartphone se demoram no comportamento de checagem do aparelho, repetindo inúmeros intervalos de checagem ao longo do dia, de modo a perder a noção do tempo de uso diário (Deng et al., 2019). Percebeu-se, conforme os resultados (figura 3), que 94,07\% dos indivíduos com altos níveis de dependência de Smartphone tiveram a impressão de que o intervalo da atividade proposta na pesquisa "demorou um pouco" ou "demorou muito", de modo que nenhum $(0.0 \%)$ considerou que a passagem do tempo tenha se dado de forma "normal" ao longo da atividade. Em contrapartida, 70,80\% dos indivíduos sem dependência marcaram que a atividade "demorou um pouco" ou "demorou muito", tendo pelo menos $24,40 \%$ considerado que o tempo transcorreu de forma "normal". Essa diferença significativa indica que a presença do fator de alta dependência entre os grupos produziu uma distribuição diferente das frequências, não justificada pelo baixo grau de hedonismo experimentado na atividade, que foi homogênea nos dois grupos (figura 4). Ademais, a neuroquímica cerebral tem influência no processamento do tempo (Meck, 1996), de modo que o relógio interno do organismo, usado para a percepção de duração do tempo na escala de minutos, está diretamente associado às funções da dopamina e do glutamato, na sua ação de regulação da percepção temporal no córtex pré-frontal (Cheng, Ali \& Meck, 2007; Radua et al., 2014)

Considerando aspectos neuropsicológicos ligados à percepção do tempo, tem-se indícios, através de estudos de neuroimagem funcional, de que as funções executivas e de controle cognitivo, como a atenção (Barrientos, Restrepo, \& Arbeláez, 2016), compartilham a mesma base funcional e neuroanatômica que a percepção temporal (Radua, Del Pozo, Gómez, GuillénGrima \& Ortuño, 2014). Suscita-se que a superestimação do tempo em usuários massivos de Smartphone ocorre devido à limitação e a sobrecarga cognitiva no processamento de informações na consciência (Lang, 2000), graças ao uso simultâneo e alternado de diversas atividades executivas (leitura, escrita, visualização de imagens e vídeos, funções motoras do teclado, etc.), próprios da interação fragmentada com o Smartphone e seus diversos aplicativos (Deng et al., 2019). O alto processamento de informações e estímulos provoca a impressão de que o tempo se dilata, parecendo maior do que realmente é, havendo proporcionalidade entre o aumento da percepção de estímulos e a sensação de dilatação temporal (Glicksohn, 2001), em decorrência da maior atividade das funções cognitivas, como atenção, memória e percepção (Matthews \& Meck, 2016).

De outro modo, pode-se acrescentar que as diferenças na percepção do tempo podem ocorrer devido aos níveis de satisfação obtidas na atividade desempenhada, que funciona como uma recompensa comportamental, influenciando a percepção temporal (Hornik, 1984). Porém, o sistema de gratificação sofreria alterações conforme o estabelecimento de um quadro de dependência (Picon et al., 2015). Levando isso em conta, deve-se considerar também as alterações neuroplásticas inerentes a 
dependências comportamentais, como no caso de tecnologia/Smartphone, que envolvem alteração nos neurotransmissores do circuito de recompensa, como a dopamina, que também cumpre função na construção da percepção do tempo dos estímulos (Cheng, Ali \& Meck, 2007), encaminhando-se para o uso compulsivo a partir da tolerância desenvolvida ao comportamento de uso (Oliveira, 2012). Portanto, o uso de Smartphone, além de alterações cognitivas nos mecanismos de memória e de atenção, alteraria também os mecanismos de gratificação e de obtenção de recompensa dos indivíduos (Wilmer, Sherman, \& Chein, 2017).

A dispersão altíssima das estimativas de tempo do grupo com alta dependência em torno da média (figura 2) revela que há a possibilidade de que a atenção empregada por um número destes participantes tenha sido tão dispersa que a estimativa final foi feita de maneira arbitrária, o que explicaria a presença de valores extremamente baixos e extremamente altos neste grupo. Este fenômeno pode ser semelhante ao encontrado no enviesamento de respostas, como os de desejabilidade social presentes em auto-relatos, onde o participante da pesquisa pode enviesar sua resposta para se adequar à uma imagem social considerada positiva (Gouveia, Guerra, Sousa, Santos \& Mesquita Costa, 2009). Pelo caráter neutro do estímulo apresentado neste estudo, é improvável que um possível enviesamento tenha ocorrido devido à desejabilidade social. Entretanto, uma motivação mais plausível para enviesamento neste contexto seria o das respostas não contingentes ou ao acaso, onde respostas são emitidas por descuido ou desatenção - novamente corroborando a hipótese de que o uso prolongado de smartphone interfere com os sistemas de gratificação e obtenção de recompensas, além de gerar dificuldades na manutenção da atenção (Almiro, 2017).

O grupo etário com o qual a pesquisa foi feita é bastante jovem, composto por graduandos que tinham por volta de 21,5 anos de idade. Todos os participantes alegaram possuir pelo menos dois aparelhos com acesso à internet. A adesão desde cedo de novas tecnologias como a das redes sociais e do smartphone fazem com que boa parte do desenvolvimento destas pessoas tenha sido moldado por esta nova dinâmica. Estas alterações no desenvolvimento infantojuvenil ainda estão sendo exploradas por estudos recentes, mas podem ir de alterações posturais (Lima, Sousa Cabral, Cruz, Freitas \& Baracat, 2021) até consequências psicossociais (Dias, Oliveira Lyrio, Fonseca \& Rodrigues, 2019). Nem todas as consequências são negativas, alguns autores encontraram associações positivas entre o uso de tablets e celulares smartphone com o desenvolvimento infantil. As mesmas funções cognitivas supracitadas como a memória podem ser bastante desenvolvidas através da instrumentalização destas tecnologias (Santos, 2018). Entretanto, outras pesquisas apontam para o surgimento de características extremamente prejudiciais a longo prazo, principalmente aos já citados mecanismos de gratificação e recompensa e a atenção. A privação sensório-motora e sócio-afetiva causada pelo uso de mais de 4 horas por dia de ambiente virtual pode ativar comportamentos semelhantes àqueles do Transtorno do Espectro Autista - TEA (Dias et al., 2019).

O fenômeno de superestimar o tempo em intervalos curtos também pode ser observado em pessoas com transtorno de humor, como na depressão (Kent, Van Doorn \& Klein, 2019). A dependência de smartphone também está associada a comportamentos evitativos, ligados à baixa auto-estima ou à personalidade introvertida, além de outros transtornos psicopatológicos, como à ansiedade social ou fobia social, podendo causar ataques de pânico, caso o aparelho não esteja disponível para o uso em dado momento (Bhattacharya et al., 2019). A mente pode ser considerada uma propriedade que emerge da interação de diversos elementos do cérebro, de modo que a organização desses elementos neurológicos é fundamental para que todos os recursos da consciência sejam adequados, incluindo a percepção temporal (Allman, Yin \& Meck, 2014).

\section{Conclusão}

Conclui-se que o grupo com alto nível de dependência de Smartphone apresentou percepção aumentada de duração do tempo em relação ao grupo sem dependência, havendo diversos fatores que influenciam nesse fenômeno, desde alterações da ordem cognitiva e comportamental, do circuito de gratificação e recompensa, até condições que podem estar indiretamente associadas ao quadro de dependência de Smartphone, como ansiedade e depressão. Num cenário em que a tecnologia está cada vez mais presente na vida dos indivíduos modernos, é de fundamental importância identificar as alterações nas funções cognitivas 
e no cérebro humano, a nível estrutural e funcional, advindas do uso compulsivo e da dependência de tecnologia. Dessa forma, conclui-se que a percepção do tempo estrutura a velocidade e a passagem das vivências dos indivíduos, tendo papel fundamental na organização dos hábitos, nos comportamentos diários e na tomada de decisões, sendo construído a partir de um complexo arcabouço suscetível a perturbações advindas da relação com a tecnologia, em especial, com o Smartphone.

Diante da pandemia de COVID-19, houve um aumento exponencial no uso de tecnologia digital em todo o mundo, de modo a mudar profundamente os hábitos diários dos seres humanos, com influência direta nos processos cognitivos e relacionais, afetando a saúde mental dos indivíduos. Portanto, para além das benesses advindas do mundo virtual e tecnológico, cresce cada vez mais o estudo sobre os quadros de dependência de tecnologia, a partir do prejuízo na qualidade de vida e comprometimento do bem-estar dos indivíduos. A presente investigação buscou correlacionar as variáveis da percepção temporal e dos altos níveis de dependência em Smartphone, em estudantes universitários de Campina Grande - PB, através de um estudo descritivo, visando contribuir com a discussão moderna sobre os impactos da tecnologia na mente humana e na saúde mental dos indivíduos. Esperase que o presente estudo tenha favorecido o avanço acerca das alterações advindas das dependências de Smartphone na cognição humana, no que toca à percepção do tempo, cabendo a sugestão para futuras investigações desse fenômeno moderno a partir da pesquisa científica comprometida.

\section{Referências}

Allman, M. J., Yin, B., \& Meck, W. H. (2014). Time in the psychopathological mind. In. Arstila, V. \& Lloyd, D. (Eds.), Subjective time: The philosophy, psychology, and neuroscience of temporality, 637-654. Boston Review.

Almiro, P. A. (2017). Uma nota sobre a desejabilidade social e o enviesamento de respostas. Avaliação Psicológica, 16(3). https://dx.doi.org/10.15689/ap.2017.1603.ed.

Barrientos, L. A., Restrepo, C. A., \& Arbeláez, A. C. M. (2016). La Percepción del Tiempo Psicológico y su relación con medidas atencionales. Revista de Psicología Universidad de Antioquia, 8(1), 65-82.

Bhattacharya, S., Bashar, M. A., Srivastava, A., \& Singh, A. (2019). Nomophobia: No mobile phone phobia. Journal of family medicine and primary care, 8(4), e1297.

Block, R. A., \& Zakay, D. (1996). Models of psychological time revisited. Time and mind, 33(9), 171-195.

Cheng, R. K., Ali, Y. M., \& Meck, W. H. (2007). Ketamine "unlocks" the reduced clock-speed effects of cocaine following extended training: evidence for dopamine-glutamate interactions in timing and time perception. Neurobiology of learning and memory, 88(2), 149-159.

Deng, T., Kanthawala, S., Meng, J., Peng, W., Kononova, A., Hao, Q.,Zhang, Q., \& David, P. (2019). Measuring smartphone usage and task switching with log tracking and self-reports. Mobile Media \& Communication, 7(1), 3-23.

Dias, F. M. A., Oliveira Lyrio, A. C., Fonseca Wagner, Y., \& Rodrigues, D. F. (2019). As Implicações do Uso Excessivo de Smartphones e Tablets por Crianças e Jovens. Redin-Revista Educacional Interdisciplinar, 8(1).

Droit-Volet, S., Monceau, S., Berthon, M., Trahanias, P., \& Maniadakis, M. (2018). The explicit judgment of long durations of several minutes in everyday life: Conscious retrospective memory judgment and the role of affects? PLoS One, 13(4): e0195397.

Estrela, C. (2018). Metodologia Científica: Ciência, Ensino, Pesquisa. Editora Artes Médicas.

Glicksohn, J. (2001). Temporal cognition and the phenomenology of time: A multiplicative function for apparent duration. Consciousness and Cognition, 10(1), $1-25$.

Gouveia, V. V., Guerra, V. M., de Sousa, D. M. F., Santos, W. S., \& de Mesquita Costa, J. (2009). Escala de Desejabilidade Social de Marlowe-Crowne: evidências de sua validade fatorial e consistência interna. Avaliação Psicológica, 8(1), 87-98.

Hornik, J. (1984). Subjective vs. objective time measures: A note on the perception of time in consumer behavior. Journal of consumer research, 11(1), 615618.

Huckins, J. F., DaSilva, A. W., Wang, W., Hedlund, E., Rogers, C., Nepal, S. K., \& Campbell, A. T. (2020). Mental health and behavior of college students during the early phases of the COVID-19 pandemic: Longitudinal smartphone and ecological momentary assessment study. Journal of medical Internet research, 22(6), e20185.

Kent, L., Van Doorn, G., \& Klein, B. (2019). Time dilation and acceleration in depression. Acta psychologica, 194, 77-86.

Kononowicz, T. W., Van Rijn, H., \& Meck, W. H. (2018). Timing and time perception: A critical review of neural timing signatures before, during, and after the to-be-timed interval. Stevens' handbook of experimental psychology and cognitive neuroscience, 1, 1-38. 
Lang, A. (2000). The limited capacity model of mediated message processing. Journal of communication, 50(1), 46-70.

Lima, C. O., Sousa Cabral, L., Cruz, L. R., Freitas, T. C. B., \& Baracat, P. J. F. (2021). Interferências posturais ocasionadas pela utilização de smartphones na fase infantojuvenil. Biológicas \& Saúde, 11(36), 55-71.

Matthews, W. J., \& Meck, W. H. (2016). Temporal cognition: Connecting subjective time to perception, attention, and memory. Psychological bulletin, 142(8), e865.

Meck, W. H. (1996). Neuropharmacology of timing and time perception. Cognitive brain research, 3(3-4), 227-242.

Nather, T. C., Fernandes, P. A. M., \& Bueno, J. L. O. (2014). Subjective time perception is affected by different durations of exposure to abstract paintings that represent human movement. Psychology \& Neuroscience, 7(3), 381-392.

Oliveira, A. F. P. (2012). Neurobiologia dos comportamentos aditivos, Mestrado Integrado em Medicina, Faculdade de Medicina - Universidade do Porto. Portugal - Porto.

Pereira A. S. et al. (2018). Metodologia da pesquisa científica. UFSM.

Picon, F., Karam, R., Breda, V., Restano, A., Silveira, A., \& Spritzer, D. (2015). Precisamos falar sobre tecnologia: caracterizando clinicamente os subtipos de dependência de tecnologia. Revista brasileira de psicoterapia, 17(2), 44-60.

Radua, J., Del Pozo, N. O., Gómez, J., Guillen-Grima, F., \& Ortuño, F. (2014). Meta-analysis of functional neuroimaging studies indicates that an increase of cognitive difficulty during executive tasks engages brain regions associated with time perception. Neuropsychologia, 58, 14-22.

Rammsayer, T. H., Borter, N., \& Troche, S. J. (2015). Visual-auditory differences in duration discrimination of intervals in the subsecond and second range. Frontiers in psychology, 6, e1626.

Ramos, D., \& Bueno, J. L. O. (2012). Emoções de uma escuta musical afetam a percepção subjetiva de tempo. Psicologia: Reflexão e Crítica, 25 , $286-292$.

Sales, H. F. S., Silva, F. M. S. M., Lopes, B. J., \& Silva, C. F. L. S. (2018). Adaptação da escala de uso compulsivo de Internet para avaliar dependência de smartphone. Avances en Psicología Latinoamericana, 36(1), 155-166.

Santos, L. R. (2018). A influência da utilização de mídias interativas (tablet e smartphone) no desenvolvimento cognitivo e de linguagem em crianças de 24 a 42 meses. Mestrado em Ciências Fonoaudiológicas, Faculdade de Medicina - Universidade Federal de Minas Gerais. Brasil - Belo Horizonte.

Sinnett, S., Jäger, J., Singer, S. M., \& Antonini Philippe, R. (2020). Flow states and associated changes in spatial and temporal processing. Frontiers in psychology, 11,381 .

Tejedor, S., Cervi, L., Pérez-Escoda, A., \& Tusa, F. (2020). Smartphone usage among students during COVID-19 pandemic in Spain, Italy and Ecuador. In. Eighth International Conference on Technological Ecosystems for Enhancing Multiculturality, 571-576.

Tonelli, H. (s.d) Introdução à psicopatologia e à neurobiologia das adições e dependências químicas e comportamentais. ACADEMIA.edu. Disponível em <<(PDF) Introdução à Psicopatologia e à Neurobiologia das Adições e Dependências Químicas e Comportamentais | Hélio Tonelli - Academia.edu>>

Wilmer, H. H., Sherman, L. E., \& Chein, J. M. (2017). Smartphones and cognition: A review of research exploring the links between mobile technology habits and cognitive functioning. Frontiers in psychology, 8, 605.

Worms, F. (2004). A concepção bergsoniana do tempo. Dois Pontos, 1(1).

Zakay, D. (1990). The evasive art of subjective time measurement: Some methodological dilemmas. In. Block, R. A. Cognitive models of psychological time, 59-84. Lawrence Erlbaum Associates, Inc. 